\title{
Zika - The road from an obscure disease to an epidemic of information
}

\author{
Flavia Masson de Moraes ${ }^{[1]}$, Danillo Lucas Alves Esposito ${ }^{[1]}$ \\ and Benedito Antônio Lopes da Fonseca ${ }^{[1]}$
}

\begin{abstract}
[1]. Departamento de Clínica Médica, Faculdade de Medicina de Ribeirão Preto, Universidade de São Paulo, Ribeirão Preto, SP, Brasil.
\end{abstract}
Zika virus (ZIKV) infections have been increasingly reported in many countries of tropical and subtropical regions of the world where infestation with the vector, Aedes aegypti, and possibly, Aedes albopictus, contribute to the spread of the infection. ZIKV infections, when symptomatic, cause a mild exanthematic disease named Zika. In addition to vector transmission, recent reports have uncovered other forms of ZIKV transmission - congenital, sexual, and through blood/ blood component transfusion ${ }^{1}$. Although Zika has increased its incidence recently, the ZIKV was first isolated in Uganda in 1947 from the blood of an infected Rhesus monkey that was used as a sentinel in research on the yellow fever virus ${ }^{2}$. However, it was only after an epidemic in the Yap island, Federated States of Micronesia, in 2007, that the disease was clinically distinguished from dengue by physicians and healthcare workers involved in the investigation of the epidemic ${ }^{3}$. Public health authorities worldwide have only turned their attention to Zika in the past years due to the increasing number of reported infections (mostly throughout the Americas) and the complications associated with the disease [microcephaly in neonates and Guillain-Barré syndrome (GBS)].

ZIKV is a flavivirus that shares common antigenicity with other viruses from the Flaviviridae family, in particular, the dengue virus. Both diseases present with similar clinical manifestations after infection, complicating the clinical diagnosis of Zika. Moreover, this antigenic similarity results in a significant cross-reactivity of antibodies elicited by both infections. This is more evident for immunoglobulin G (IgG) antibodies (convalescent phase of the disease) when compared to IgM antibodies (acute phase of the disease).

Although most Zika cases are believed to be asymptomatic, the symptoms of ZIKV infection are typically described as a mild form of dengue, mainly characterized by a low-grade fever, myalgia, arthralgia, headache, pruritic maculopapular rash, and non-purulent conjunctivitis ${ }^{4}$. Severe cases are uncommon,

Corresponding author: Dr. Benedito Antônio Lopes da Fonseca. e-mail: baldfons@fmrp.usp.br

Received 30 December 2016

Accepted 7 February 2017 but the increasing incidence of microcephaly in newborns born to women who were infected with the ZIKV during their pregnancies and the increasing number of cases of GBS occurring concomitantly with Zika outbreaks has created a global concern around this illness. The incidence of microcephaly, a condition in which the head circumference of a newborn is smaller than normal, increased in the months following a large Zika outbreak that started in 2015 in Northeastern Brazil and spread nation-wide in 2016. Microcephaly can lead to a severe cognitive delay and is typically associated with other problems such as motor and visual impairment ${ }^{5}$.

Another neurological complication of ZIKV infection is GBS. This disorder is characterized by a demyelination of motor axons, typically as a result of an autoimmune response that might be triggered by several mechanisms, including infection by several pathogens ${ }^{6}$. The association between GBS and ZIKV infection was first described after a Zika outbreak in French Polynesia outbreak. The same association was observed in Brazil after the 2015/2016 epidemic. The molecular mechanism linking ZIKV infection to GBS is unknown. Several studies are being performed to investigate this association. Similar to the dengue and chikungunya diseases, no treatment or specific vaccine exist for Zika. Current treatment only targets symptoms and typically includes drugs used to reduce fever and pain.

It is believed that the ZIKV was introduced to Brazil in the second half of $2013^{7}$; since 2015, autochthonous transmissions of ZIKV have been reported in Brazil and other Central and South American countries. The Brazilian Ministry of Health estimates that, in 2016, 200,465 cases of ZIKV infection occurred until September 17; of those, 109,596 were confirmed by a clinical or epidemiological aspects ${ }^{8}$, as no specific laboratory test to accurately diagnose $\mathrm{Zika}$ exists. The geographic region in Brazil that has shown the highest Zika incidence in 2016 was the Midwest (193.5 cases/100,000 inhabitants). Three deaths were confirmed in 2016: two in Rio de Janeiro and one in Espírito Santo State ${ }^{8}$. A highly susceptible population (which have never been infected by the virus), favorable climate, and the widespread presence of Aedes mosquitoes in the entire country might have contributed to the fast spread of the disease. 
Most of what we know about Zika is based on data from three important outbreaks (Micronesian island of Yap in 2007, French Polynesia and New Caledonia in 2013/14, and Brazil from 2014 onwards). Due to the large number of complications associated with ZIKV infection that were reported after the Brazilian outbreak, the clinical-epidemiological outcome of the disease has been changing constantly; this, in turn, resulted in an increasing number of studies on ZIKV infection, including those on epidemiology, virus infectivity, its interaction with other pathogens and diseases, the molecular mechanisms occurring after infection, and therefore those studies could help in the diagnosis of the disease, and vaccine development.

In this issue of the Journal of the Brazilian Society of Tropical Medicine, the article entitled Zika virus, from discovery to the present days: Bibliometric review study describes extensive bibliographic research and summarizes all articles on ZIKV infection that were published between January 1947 and May 2016. Although it does not bring any novelty to the field of ZIKV research, this bibliographic review describes the most important features of the disease. The authors considered the publication language of the included articles and the research approaches that were being utilized. They selected 321 publications of five different databases, including LILACS (Literatura LatinoAmericana e do Caribe em Ciências da Saúde), Scientific Electronic Library Online (SciELO), Biblioteca Virtual em Saúde (BVS), MEDLINE and PubMed, based on predetermined inclusion criteria.

The study presents an enormous compilation of data that were described in ZIKV-related publications. It is, therefore, a useful tool to enlighten oneself with all information (included in some of the most reliable online databases for scientific research in countries with ZIKV infections) that is available to date. The authors emphasize that until 2007, ZIKV infection was not a high demand concern in the scientific community. This changed that after 2007 when the number of reported cases increased significantly. As a result, the number of publications and research on the ZIKV, its disease and complications have reached new levels. The concerns regarding pregnant women and microcephaly in newborns has contributed significantly to the increase in research on this disease and as a result, the number of publications.

Although the number of research on ZIKV infections and its complications have increased within the past two years, more research is needed to elucidate the mechanisms causing more severe disease outcomes, such as GBS and microcephaly, establish strategies to prevent ZIKV infections and complications of the disease, and improve the sensitivity of Zika diagnosis in countries such as Brazil, where a great number of people have been exposed to other flavivirus infections. Diagnostic sensitivity is particularly important during the convalescent phase of the disease, since Zika symptoms are very similar to those of other flavivirus-related diseases, with a very short viremia and a high antibody cross-reactivity in serological tests.

The review on ZIKV infections will help readers who are not familiar with web searches of research publications familiarize themselves with a large amount of data on ZIKV infections that have been published to date.

\section{REFERENCES}

1. Musso D, Roche C, Robin E, Nhan T, Teissier A, Cao-Lormeau VM. Potential sexual transmission of Zika virus. Emerg Infect Dis. 2015; 21:359-361

2. Simpson DI. Zika virus infection in man. Trans R Soc Trop Med Hyg. 1964; 58:335-338. doi: 10.1016/0035-9203(64)90201-9.

3. Duffy MR, Chen TH, Hancock WT, Powers AM, Kool JL, Lanciotti RS, et al. Zika virus outbreak on Yap Island, Federated States of Micronesia. N Engl J Med. 2009; 360(24):2536-43.

4. Shuaib W, Stanazai H, Abazid AG, Mattar AA. The reemergence of Zika virus: a review on pathogenesis, clinical manifestations, diagnosis, and treatment. Am J Med. 2016; doi: 10.1016/j. amjmed.2016.02.027.

5. Centers for Disease Control and Prevention (CDC). National Birth Defects Prevention Network. Major Birth Defects Data from Population-based Birth Defects Surveillance Programs in the United States, 2006-2010. Birth Defects Research. (Part A) 2013; 97:S1-S172.

6. Hughes RA, Cornblath DR. Guillain-Barre syndrome. Lancet. $2005 ; 366: 1653-1666$.

7. Vogel G. Don't blame sports for Zika's spread. Science. 2016; 351:1377-1378

8. Ministério da Saúde. Secretaria de Vigilância em Saúde. Monitoramento dos casos de dengue, febre de chikungunya e febre pelo vírus Zika até a Semana Epidemiológica 37, 2016. Brasília: Boletim Epidemiológico, volume 47, $\mathrm{n}^{\circ}$ 34, 2016. Disponível em: http:// http://portalsaude.saude.gov.br/images/pdf/2016/ outubro/18/2016-029-Dengue-publicacao-n-34.pdf. 